Jean Paul Obame Engone ${ }^{1}$ Louis BÉLANGER ${ }^{2}$

Séraphin ASSAME ${ }^{1}$

${ }^{1}$ École nationale des eaux et forêts

Département

Exploitation forestière

BP 3960, Libreville Gabon

${ }^{2}$ Université Laval

Département des sciences du bois et de la forêt

Pavillon Abitibi-Price, bureau 2171

2405, rue de la Terrasse Québec (Québec) G1V oA6 Canada

\section{Régime de chablis et régénération des arbres dans la Réserve de biosphère d'Ipassa au Gabon}

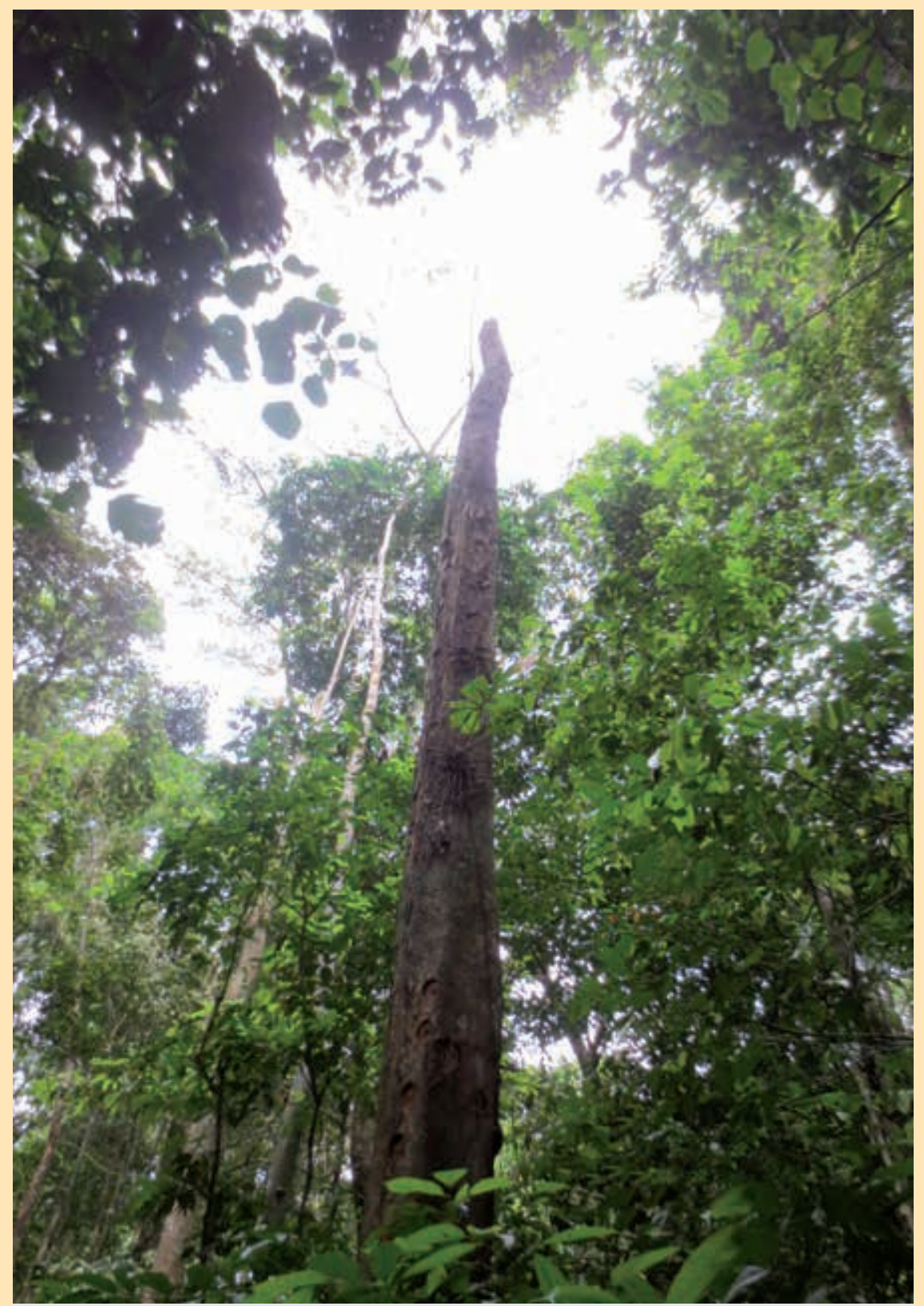

Photo 1.

Ouverture de la canopée créée par bris de la couronne d'un arbre. Photo J. P. Obame Engone. 


\section{RÉSUMÉ}

\section{RÉGIME DE CHABLIS ET RÉGÉNÉRATION DES ARBRES DANS LA RÉSERVE DE BIOSPHĖRE D'IPASSA AU GABON}

La présente étude vise à caractériser le régime de perturbations par chablis de la forêt d'Ipassa (Gabon) et à analyser les liens entre les types de chablis et la régénération des espèces d'arbres. Nous avons étudié le régime de perturbation par chablis dans un dispositif permanent de 38 ha. Le suivi annuel de nouveaux chablis a été mené durant cinq années entre 2005 et 2009. Nous avons également évalué la régénération naturelle des espèces d'arbres au sein des différents types de chablis. L'étude montre que la forêt d'Ipassa est principalement affectée par un gradient de chablis partiels et chablis élémentaires qui constituent $72 \%$ du total de chablis recensés. Ces chablis ont annuellement occupé $1,3 \%$ de la surface étudiée. La taille moyenne des chablis varie de $52 \pm 13 \mathrm{~m}^{2}$ à $625 \pm 220 \mathrm{~m}^{2}$, alors que la demi-vie est d'environ 53 ans. L'analyse des correspondances multiples montre que la régénération de 47 espèces $(25,7 \%)$ est associée aux différents types de chablis. Douze espèces d'arbres héliophiles se régénèrent exclusivement dans les chablis multiples et complexes alors que la régénération de 35 espèces tolérantes à l'ombrage se produit préférentiellement dans les chablis partiels et élémentaires. Cependant, plusieurs espèces d'arbres n'ont pas de liens manifestes avec les chablis. Cela suggère qu'en dehors des chablis, d'autres facteurs comme la limitation en dispersion agissent sur la régénération des espèces d'arbres.

Mots-clés : espèces sciaphiles, régénération naturelle, régime de chablis, forêt tropicale, Gabon.

\section{ABSTRACT}

\section{WINDTHROW PATTERN AND REGENERATION OF TREES IN THE IPASSA BIOSPHERE RESERVE IN GABON}

This study was conducted to characterise the pattern of disturbance caused by windthrow in the forest of Ipassa (Gabon) and to analyze the links between types of windthrow and the regeneration of different tree species. The pattern of windthrow disturbance was studied in a permanent 38 hectares plot. Windthrow was monitored annually for five years from 2005 to 2009 . We also assessed the natural regeneration of trees according to different types of windthrow. The study results show that $72 \%$ of windthrown trees in the forest of Ipassa are partially fallen or single trees. Each year, these occupied $1.3 \%$ of the surface area studied. The scale of windthrow varied on average from $52 \pm 13 \mathrm{~m}^{2}$ to $625 \pm 220 \mathrm{~m}^{2}$, with a half-life of about 53 years. Multiple Correspondence Analysis showed that in 47 species $(25.7 \%)$, regeneration is associated with the different types of windthrow. Twelve species of sun-loving trees regenerate exclusively in multiple or complex windthrow situations, while 35 shade-tolerant species tend to regenerate in partial and single-tree windthrow situations. In several tree species, there is no obvious link between regeneration and windthrow patterns. This suggests that other factors besides windthrow, such as limited dispersal, influence the regeneration of tree species.

Keywords: sciaphilous species, natural regeneration, pattern of windthrow, tropical forest, Gabon.

\section{RESUMEN}

\section{RÉGIMEN DE DESARRAIGO Y REGENERACIÓN DE ÁRBOLES EN LA RESERVA DE LA BIOSFERA DE IPASSA EN GABÓN}

El objetivo de este estudio es caracterizar el régimen de perturbaciones por descuaje en el bosque de Ipassa (Gabón) y analizar las relaciones entre tipos de descuaje y regeneración de especies de árboles. Se estudió dicho régimen de perturbaciones mediante un diseño permanente de 38 ha. Durante cinco años, de 2005 a 2009 , se efectuó el seguimiento anual de los nuevos casos. Asimismo, se evaluó la regeneración natural de las especies de árboles en los distintos tipos de descuaje. El estudio muestra que el bosque de Ipassa está afectado principalmente por un gradiente de descuajes parciales y completos que suponen el $72 \%$ del total de casos registrados. La superficie anual afectada cubre el 1,3\% del área estudiada. El tamaño promedio de los chablis oscila entre $52 \pm 13 \mathrm{~m}^{2}$ y $625 \pm 220 \mathrm{~m}^{2}$, mientras que la vida media es de unos 53 años. El análisis de correspondencias múltiples muestra que la regeneración de 47 especies (25,7\%) está asociada a los distintos tipos de descuaje. Doce especies de árboles heliófilos se regeneran exclusivamente en descuajes múltiples y complejos, mientras que la regeneración de 35 especies umbrófilas se desarrolla sobre todo en los descuajes parciales y completos. No obstante, varias especies de árboles no muestran relaciones claras con los descuajes. Esto parece indicar que, además del descuaje, hay factores como la limitación de la dispersión que intervienen en la regeneración de las especies de árboles.

Palabras clave: especies esciáfilas, regeneración natural, régimen de desarraigo, bosque tropical, Gabón. 


\section{Introduction}

Les forêts gabonaises comptent parmi les forêts africaines les plus riches en biodiversité. Par rapport à celles des autres pays du Bassin du Congo, elles subissent moins de pressions en raison de la faible densité de la population (Laurance et al., 2006). Le taux net de dégradation annuelle du couvert végétal est de $0,08 \%$ pour une moyenne régionale de 0,15\% ( \pm 0,03\%) par an (Duveiller et al., 2008). À l'instar d'autres pays d'Afrique centrale, le Gabon a adopté une nouvelle loi forestière et des normes de gestion durable des forêts (Drouineau et Nasi, 1999 ; ATIBT-FFEM, 2014). La mise en œuvre de la gestion durable nécessite une bonne compréhension de la dynamique forestière.

Or, en forêt dense tropicale, les chablis constituent un des moteurs de la dynamique forestière (Oldeman, 1979 ; Riera et Alexandre, 1988). Les théories sur les chablis présument ainsi que la mort d'un arbre de la canopée par chablis crée une trouée dans le couvert forestier et initie une séquence de successions (Yamamoto, 1992 ; Denslow et al., 1998 ; Schnitzer et al., 2008). La reconstitution de la trouée se déroule selon une suite de phases (gap, building, mature) qui débute avec les espèces pionnières et évolue vers la dominance d'espèces tolérantes à l'ombrage (Rollet, 1969 ; Mutoji-A-Kazadi, 1977 ; Hartshorn, 1978 ; Florence, 1981 ; Rollet, 1983 ; Oldeman, 1989 ; Richards, 1996).

Les travaux de plusieurs auteurs ont ainsi établi que les chablis déterminent la régénération et le maintien des arbres héliophiles dans les peuplements forestiers (Brokaw, 1987 ; Sherman et al., 2000 ; Schnitzer et Carson, 2001 ; Martins et Rodrigues, 2002). Clark et Clark (1992), Zanne et Chapman (2005) ont pour leur part rapporté, s'agissant des forêts tropicales du Costa Rica et d'Ouganda, l'installation et le maintien d'espèces tolérantes à l'ombrage dans les trouées de chablis. Cependant, Clark et al. (1993) et Hubbell et al. (1999) soutiennent que les espèces tolérantes à l'ombrage qui constituent la majorité des arbres de la forêt tropicale ne montrent pas de préférence pour s'établir dans les trouées de chablis.

Pour des opinions si contrastées, on constate qu'un facteur important est insuffisamment considéré : la variabilité des types de chablis. Comme le suggèrent Denslow (1980), Orians (1982), Denslow (1987) et Brokaw (1987), la variabilité des milieux générée par les différents types de chablis devrait accommoder une large gamme d'espèces d'arbres. Certaines espèces auraient une préférence pour les chablis de petite taille et d'autres pour les grands chablis. Justement, en forêt tropicale humide d'Ipassa au Gabon, Florence (1981) a décrit plusieurs types de chablis dont les chablis partiels, les chablis élémentaires, les chablis multiples et les chablis complexes. Ce gradient de chablis créant différents niveaux d'ouverture du couvert pourrait fournir des explications sur la présence dans les peuplements forestiers d'espèces héliophiles et d'autres espèces tolérantes à l’ombrage. Florence (1981) a principalement analysé la sylvigenèse dans les chablis élémentaires. Ses travaux ont mis en évidence une différence entre les pôles proximal (surface de projection au sol de l'ancien houppier) et distal (lieu de chute des branches) des chablis élémentaires. La régénération serait freinée, voire empêchée dans le pôle distal par les branchages et débris organiques, alors que le pôle proximal offre un microsite plus favorable (butte de déracinement, meilleures conditions de lumière). L'auteur a par ailleurs relevé des cas de blocage de la reconstitution des trouées liés à la surabondance des lianes.

Malgré ces avancées, des lacunes persistent sur le rôle des chablis dans la forêt d'Ipassa. Très peu d'informations sont disponibles sur celui que joue chaque type de chablis chez les espèces tolérantes à l'ombrage. On ignore également les liens entre les chablis multiples, les chablis complexes et les espèces d'arbres. Dans la présente étude, nous avons cherché à répondre aux questions suivantes: comment s'exprime la variabilité des types de chablis et le régime de perturbations par chablis dans la forêt d'Ipassa? Est-ce que la régénération des espèces d'arbres est associée préférentiellement aux différents types de chablis ? Notre étude explore le rôle possible des types de chablis comme facteurs déterminant la présence et le maintien d'espèces d'arbres dans les peuplements forestiers, particulièrement les espèces tolérantes à l'ombrage.

Nous avons principalement visé, d'une part, à caractériser le régime de perturbations par chablis (fréquences, superficies, proportion de surface affectée, temps de renouvellement) et, d'autre part, à analyser les liens entre les types de chablis et la composition de la régénération des espèces d'arbres. Nous avons testé l'hypothèse selon laquelle le régime de perturbation par chablis de la forêt d'Ipassa était caractérisé par la dominance d'un gradient de chablis de faible dimension qui favoriseraient la régénération des espèces d'arbres tolérantes à l'ombrage.

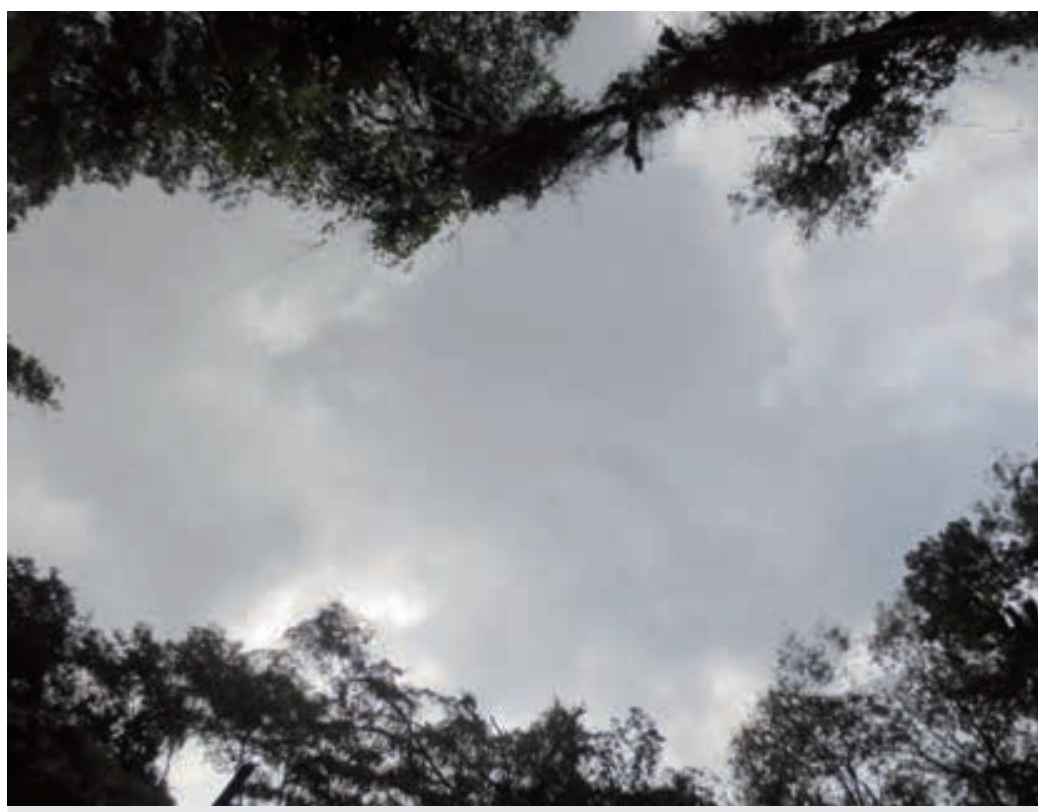

Photo 2.

Ouverture de la canopée créée par un chablis multiple.

Photo J. P. Obame Engone. 


\section{Matériel et méthodes}

\section{Site d'étude}

L'étude a été conduite dans la Réserve de biosphère d'Ipassa. Cette réserve se situe à $0^{\circ} 34^{\prime} 51^{\prime \prime}$ de latitude Nord et $12^{\circ} 50^{\prime} 22^{\prime \prime}$ de longitude Est au Nord-Est du Gabon, à près de $8 \mathrm{~km}$ au Sud de la ville de Makokou. Elle couvre une superficie d'environ 10 ooo hectares. Classée Réserve de biosphère en 1983, la forêt d'Ipassa n'a jamais été soumise à l'exploitation industrielle du bois. Elle comporte l'une des dernières forêts encore intactes du Gabon, où les processus écologiques se déroulent sans perturbations humaines. La Réserve de biosphère d'lpassa se trouve dans la " région naturelle des Plateaux du Nord et du Nord-Est » (Cabalé et Fontès, 1978). Elle est caractérisée par une forêt dense humide sempervirente à Scyphocephalium ochocoa Warb., Pycnanthus angolensis (Welw.) Warb., Pentaclethra eetveldeana (De Wild.) T. Durand, Celtis tessmannii Rendle, Gilletiodendron pierreanum (Harms) J. Léonard et Gilbertiodendron dewevrei (De Wild.) J. Léonard. Ces groupes d'espèces peuvent s'accompagner de Scorodophloeus zenkeri Harms, Santiria trimera (Oliv.) Aubrév., Panda oleosa Pierre, Fagara macrophylla (Oliv.) Engl., Petersianthus macrocarpus (P. Beauv.) Liben et Baillonella toxisperma Pierre.

Le climat est de type équatorial avec deux saisons sèches et deux saisons des pluies. La pluviosité moyenne annuelle est de $1700 \mathrm{~mm}$. La température moyenne annuelle est de $23,9^{\circ} \mathrm{C}$ avec un minimum mensuel en juillet de $21,6^{\circ} \mathrm{C}$ et un maximum en mai de $25,3^{\circ} \mathrm{C}$. L'humidité relative de l'air est très élevée, avec une moyenne de $85 \%$ (Iret-Ecotrop et Unesco, 1987).

La géologie présente en majorité des roches métamorphiques du précambrien de base (Beaujour, 1971) et la géomorphologie du site se caractérise par une succession de plateaux et de vallées. Les sols sont essentiellement composés de ferralsols xanthiques qui occupent les plateaux et les bas de pente. Les fonds de vallée sont couverts de gleysols, alors que des fluvisols sont observés sur les bords de l'Ivindo (Van Kekem, 1984).

\section{Échantillonnage}

\section{Inventaire initial des arbres}

Les travaux ont été conduits sur 38 ha au sein de la zone de recherche. Cette partie de la réserve (environ 150 ha) est quadrillée par un système de coordonnées de repérage matérialisé au sol à l'aide d'un réseau de transects permanents espacés de $100 \mathrm{~m}$, traçant ainsi des quadrats contigus de $100 \mathrm{~m} \times 100 \mathrm{~m}$. En 2005, au début de l'étude, nous avons procédé dans l'ensemble de la surface étudiée au recensement par sondage en plein des arbres de diamètre supérieur ou égal à $40 \mathrm{~cm}$. Pour chaque arbre vivant, nous avons réalisé l'identification des espèces et la mesure de diamètre à hauteur de poitrine $(1,30 \mathrm{~m})$.

\section{Inventaire des chablis}

Dans cette étude, le terme chablis désigne aussi bien une grosse branche, la couronne d'un arbre ou un arbre entier tombé au sol que la perturbation qui lui est associée. Nous avons adopté une typologie fonctionnelle des chablis adaptée des travaux de Florence (1981) et Riera et al. (1988). Cette typologie exprime l'origine et la nature du chablis et distingue le chablis partiel (chute d'une branche ou bris du houppier d'un arbre), le chablis élémentaire (chute d'un seul arbre), le chablis multiple (chute simultanée de plusieurs arbres) et le chablis complexe (chutes de plusieurs arbres, décalées dans le temps). Cette typologie avait déjà été validée en forêt gabonaise par Florence (1981).

Nous avons effectué l'inventaire des chablis en procédant à un sondage en plein sur 38 ha dans des quadrats de $100 \mathrm{~m} \times 100 \mathrm{~m}$. Le recensement des chablis a été réalisé au cours des années 2005, 2006, 2007, 2008 et 2009. Plusieurs équipes composées chacune de quatre personnes (un botaniste et trois techniciens de la station de recherche d'Ipassa) parcouraient la surface de chaque quadrat en étant alignés et en évoluant d'est en ouest. Lorsqu'un chablis était identifié, nous avons mesuré les paramètres de la trouée qui suivent : le type de chablis, l'arbre initiateur et la dimension de la trouée. Les types de chablis ont été évalués en se référant aux caractéristiques décrites précédemment. Pour chaque arbre initiateur de la trouée, nous avons identifié l'espèce, mesuré le diamètre à 1,30 m, la longueur totale de l'arbre et le type de dommage. Tous les arbres de diamètre supérieur à $20 \mathrm{~cm}$ emportés par l'arbre initiateur de trouée ont également été mesurés. La dimension de chaque trouée a été mesurée avec les méthodes décrites par Runkle (1992) : une paire de lignes est localisée de manière à ce que la première tienne sur la plus grande longueur de la trouée $(\mathrm{L})$ et la deuxième, orthogonale à la première, relie les deux extrémités de la plus petite longueur de la trouée (W). Nous avons estimé la surface de la trouée comme étant égale à nLW/4 (Runkle, 1981 ; Battles et al., 1996). Lorsqu'une trouée était irrégulière, nous avons mesuré la longueur de chaque segment de ligne, de l'intersection à la bordure de la trouée, en partant de la plus grande et dans le sens des aiguilles d'une montre. Les distances du centre vers les bords ont été mesurées selon au moins huit directions. La surface résultante du polygone a été calculée directement en prenant la somme des quatre quarts d'ellipses déterminés par les segments de lignes ainsi délimités.

\section{Inventaire de la régénération dans les trouées}

Entre mai et décembre 2009, nous avons réalisé un inventaire de la régénération dans l'ensemble des trouées inventoriées de 2005 à 2009. Au sein de chaque trouée, nous avons recensé, dans des bandes de quadrats contigus de $1 \mathrm{~m} \times 1 \mathrm{~m}$, les individus de plus de $10 \mathrm{~cm}$ de hauteur. En deçà de cette taille, la régénération subit une forte mortalité et témoigne d'un très faible taux de recrutement (Brown et Whitmore, 1992 ; Chapman et al., 1999). Les bandes de quadras contigus de $1 \mathrm{~m} \times 1 \mathrm{~m}$ (espacées de 4,5 m) étaient disposées orthogonalement à l'axe principal de la trouée, partant d'une bordure à l'autre (figure 1). 


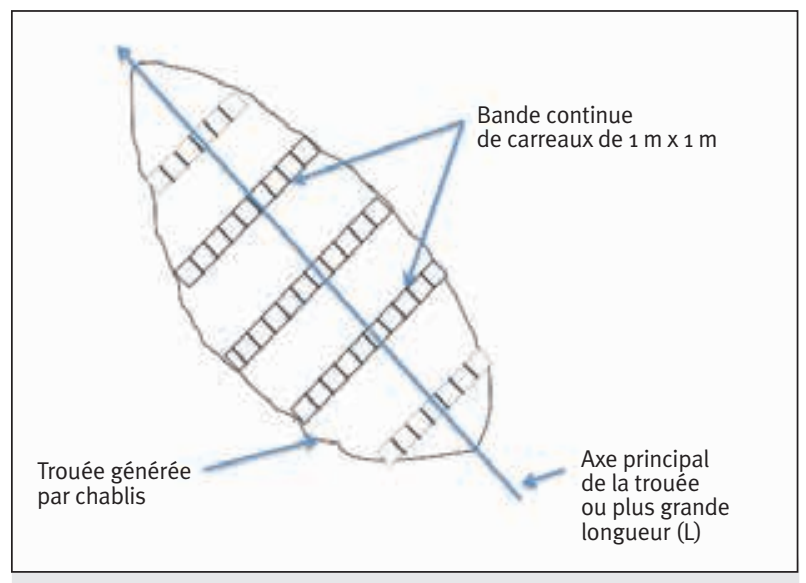

Figure 1.

Dispositif d'échantillonnage de la régénération dans les trouées : quadrats contigus de $1 \mathrm{~m} \times 1 \mathrm{~m}$.

\section{Analyses statistiques}

\section{Calcul des paramètres des trouées et du temps de renouvellement de la forêt}

Nous avons déterminé les paramètres des trouées et le temps de renouvellement de la forêt d'Ipassa avec les approches ci-dessous décrites.

- Le pourcentage de surface par type de chablis est le ratio entre la surface de chaque type de chablis et la surface totale des chablis.

- L'occurrence des chablis est le nombre de chablis ayant eu cours pendant un temps donné, sur un territoire donné, quelle que soit leur taille.

- Le temps de renouvellement a été calculé à l'aide du modèle stochastique proposé par Riera et Alexandre (1985). Selon ce modèle, pour une forêt donnée, chaque année pendant une période donnée (dt), il disparaît une proportion constante (ds) de la surface non affectée (S) traduite par l'équation :

$-\mathrm{dS} / \mathrm{dt}=\mathrm{kS}$

le signe (-) exprimant la disparition.

On en déduit la demi-vie (délai pour que la moitié de la surface forestière soit affectée par les chablis) avec la formule suivante (Riera et Alexandre, 1988) :

$$
\mathrm{t}_{0,5}=\frac{\log (0,5)}{\mathrm{a}}
$$

a étant le pourcentage annuel de chablis observé sur le site.

La demi-vie est calculée sous l'hypothèse que l'ensemble de la surface est considéré comme vierge de chablis au moment du début des observations. Le pourcentage théorique de chablis annuels $(P)$ s'obtient quant à lui à partir d'une mesure sur un intervalle de temps plus long :

$$
a=-\frac{\log P}{t}
$$

Le taux de mortalité se définit comme étant la proportion des arbres morts par rapport aux individus vivants (Rollet, 1974). Le taux de mortalité pour les arbres morts par chablis a été calculé en se basant sur les effectifs des arbres vivants sur pied de l'inventaire de 2005 et les arbres morts par chablis recensés dans l'ensemble du site pendant la durée de l'étude.

\section{Analyse des correspondances multiples}

L'analyse des correspondances multiples (ACM) a été utilisée pour comprendre les liens entre les espèces d'arbres et les différents types de chablis. Cette méthode d'analyse permet d'évaluer en même temps les comportements de plusieurs variables et de réaliser une analyse globale du phénomène. Les précisions sur les calculs liés à l'analyse des correspondances multiples peuvent être consultées dans Lebart et al. (2006) ou Escofier et Pagès (2008). Nous avons effectué les calculs à l'aide du logiciel Spad (version 7.4) en considérant 121 chablis, 183 espèces d'arbres et quatre types de chablis. Les axes factoriels significatifs ont été déterminés selon la méthode dite des bâtons brisés (Frontier, 1976 ; Legendre et Legendre, 2012). Pour l'interprétation des axes, nous avons retenu comme variables significatives celles qui présentent une plus forte contribution absolue (supérieure à la moyenne), une contribution relative qui tend vers 1 et une valeur test supérieure (en valeur absolue) à 2 (Saporta, 2006). Nous n'avons représenté que les deux premiers axes factoriels qui résument l'essentiel du phénomène étudié.

\section{Résultats}

\section{Variations annuelles des types de chablis}

Entre juin 2005 et juin 2009, 121 chablis ont été recensés sur le site étudié. Pour les quatre principaux types de chablis qui affectent la forêt d'Ipassa, les proportions suivantes ont été observées (tableau I) : chablis partiels (49\% du total des chablis), chablis élémentaires (23\%), chablis multiples (13\%) et chablis complexes (15\%). Les deux premiers mentionnés constituaient l'essentiel des perturbations enregistrées, totalisant environ $72 \%$ des chablis. Dans l'ensemble, le nombre de chablis variait d'une année à l'autre. Cependant, la majeure partie des chablis (environ la moitié) se sont produits en 2007 et en 2009.

\section{Superficies et proportions du territoire affecté}

Les chablis partiels constitués par la chute d'une grosse branche ou d'un houppier ont créé des trouées d'une surface moyenne de $52 \pm 13 \mathrm{~m}^{2}$. Les chablis élémentaires occasionnaient des ouvertures de la canopée plus étendues, avec une surface moyenne de $321 \pm 106 \mathrm{~m}^{2}$. Les chablis complexes généraient des trouées d'une superficie moyenne de $194 \pm 72 \mathrm{~m}^{2}$, alors que la surface moyenne des chablis multiples était d'environ $625 \pm 120 \mathrm{~m}^{2}$. Le tableau II résume les 
surfaces : les proportions et la répartition des chablis pour les cinq ans d'étude. Au cours de la période d'observation, les chablis ont occupé 2,6 ha, soit $7 \%$ du territoire (environ $1,3 \%$ par an). Quoique plus fréquents, les chablis partiels n'ont couvert que $11 \%$ de la surface totale des chablis et seulement $1 \%$ des terres forestières étudiées. Les chablis élémentaires ont occupé $35 \%$ du total des surfaces couvertes en cinq ans par les chablis et près de $3 \%$ du territoire échantillonné. Les chablis multiples et les chablis complexes ont affecté près de 4 \% du territoire étudié.

\section{Mortalité par chablis}

Parmi les 7183 arbres vivants de plus de $40 \mathrm{~cm}$ de diamètre dénombrés au début de l'étude, les chablis ont causé la mort de 1101 sujets (soit $15 \%$ des effectifs). Environ $20 \%$ de ces arbres ont été déracinés. La majorité des sujets sont morts brisés. Les causes des bris d'arbres n'ont pas été déterminées. Le taux de mortalité lié aux chablis variait de 3 à $6 \%$. La figure 2 montre que la mortalité par chablis affecte toutes les classes de diamètre, aussi bien les jeunes que les vieux arbres. Les observations sur le terrain démontrent qu'en majorité, les jeunes arbres sont emportés par la chute des arbres matures.

Tableau I.

Nombre de chablis par type, recensés entre 2005 et 2009 sur 38 ha.

\begin{tabular}{|l|c|c|c|c|c|c|c|}
\hline & $\mathbf{2 0 0 5}$ & $\mathbf{2 0 0 6}$ & $\mathbf{2 0 0 7}$ & $\mathbf{2 0 0 8}$ & $\mathbf{2 0 0 9}$ & Total & $\%$ \\
\hline $\begin{array}{l}\text { Chablis } \\
\text { partiels }\end{array}$ & 9 & 11 & 14 & 10 & 15 & 59 & 49 \\
\hline $\begin{array}{l}\text { Chablis } \\
\text { élémentaires }\end{array}$ & 3 & 4 & 7 & 5 & 9 & 28 & 23 \\
\hline $\begin{array}{l}\text { Chablis } \\
\text { multiples }\end{array}$ & 2 & 2 & 4 & 2 & 6 & 16 & 13 \\
\hline $\begin{array}{l}\text { Chablis } \\
\text { complexes }\end{array}$ & 3 & 2 & 4 & 3 & 6 & 18 & 15 \\
\hline Total & 17 & 19 & 29 & 20 & 36 & 121 & 100 \\
\hline
\end{tabular}

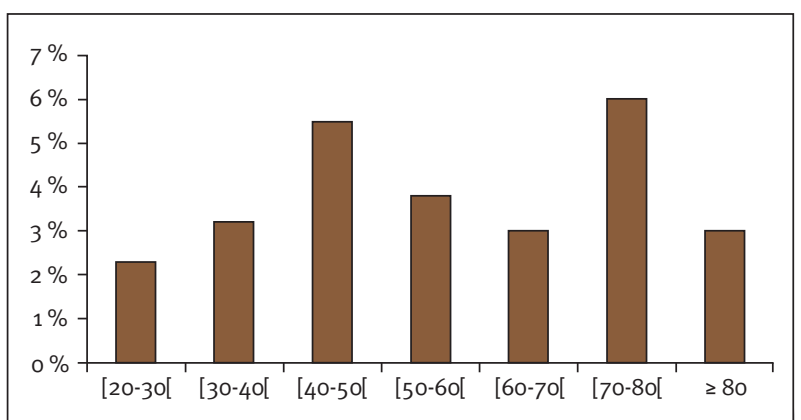

Figure 2.

Taux de mortalité associé au chablis par classe de diamètre.

\section{Temps de renouvellement de la forêt}

En moyenne, les chablis affectent annuellement environ $1,3 \%$ de la surface forestière observée. La demi-vie, ou temps pour que les chablis couvrent la moitié de la forêt, est égale à 53 ans. Le pourcentage théorique de chablis annuels pour une période de 100 ans est estimé à $4 \%$.

\section{Effet des types de chablis sur les espèces}

L'analyse des correspondances multiples fournit les résultats consignés dans les tableaux III, IV et V. Nous avons reporté dans ces tableaux les coordonnées, les contributions absolues, les contributions relatives et les valeurs tests des variables significatives associées aux axes. La méthode dite des bâtons brisés fournit trois axes significatifs à interpréter. Les variables qui ne contribuent pas significativement à la formation de ces trois premiers axes ne sont pas mentionnées dans ces tableaux. Le premier axe regroupe les chablis partiels, de très faible taille, issus de la chute de branches ou de bris de houppier et la régénération des espèces d'arbres associées qui suivent (tableau III) : Afrostyrax lepidophyllus Mildbr., Beilschmiedia congolana (Robyns) R. Wilczek, Dacryodes klaineana (Pierre) H.J. Lam, Fillaeopsis discophora

Tableau II.

Surfaces de chablis et proportions du territoire affecté par type de chablis.

\begin{tabular}{|l|c|c|c|c|c|}
\hline & $\begin{array}{c}\text { Chablis } \\
\text { complexe }\end{array}$ & $\begin{array}{c}\text { Chablis } \\
\text { multiple }\end{array}$ & $\begin{array}{c}\text { Chablis } \\
\text { élémentaire }\end{array}$ & $\begin{array}{c}\text { Chablis } \\
\text { partiel }\end{array}$ & Total \\
\hline Surface totale des chablis $\left(\mathrm{m}^{2}\right)$ & 3500 & 10000 & 9000 & 3050 & 25550 \\
\hline $\begin{array}{l}\text { Proportion surface } \\
\text { totale des chablis (\%) }\end{array}$ & 15 & 39 & 35 & 11 & 1 \\
\hline $\begin{array}{l}\text { Proportion surface totale } \\
\text { du territoire (\%) }\end{array}$ & 1 & 3 & 3 & $52 \pm 13$ & 32100 \\
\hline $\begin{array}{l}\text { Surface moyenne des trouées } \\
\text { par type de chablis }\left(\mathrm{m}^{2}\right)\end{array}$ & $194 \pm 72$ & $625 \pm 220$ & 32106 & 52 \\
\hline
\end{tabular}


Harms, Celtis tessmannii Rendle, Treculia africana Desc., Drypetes gossweileri S. Moore, Eriocoelum macrocarpum Gilg, Piptadeniastrum africanum (Hook. f.) Brenan, Dialium pachyphyllum Harms, Dacryodes buettneri (Engl.) H.J. Lam, Pancovia pedicellaris (Radlk.) Gilg, Strombosiopsis tetrandra
Engl., Strombosia pustulata Oliv., Heisteria parvifolia Sm, Campostylus mannii (Oliver) Gilg, Chytranthus angustifolius Exell, Diospyros hoyleana F. White, Diospyros conocarpa (Gürke) K. Schum., Synsepalum longecuneatum De Wild., Coelocaryon preussii Warb., Uapaca paludosa (Aubrév.)

\section{Tableau III.}

Axe 1 : chablis partiels et espèces associées.

\begin{tabular}{|c|c|c|c|c|c|c|c|}
\hline Appellation & Abréviation & Famille & Poids & Coordonnées & Contribution & $\begin{array}{l}\text { Cosinus } \\
\text { carré }\end{array}$ & $\begin{array}{c}\text { Valeur } \\
\text { test }\end{array}$ \\
\hline Chablis partiel & $\mathrm{ChP}$ & & 0,31 & 1,11 & 2,1 & 0,97 & $-9,7$ \\
\hline Afrostyrax lepidophyllus Mildbr. & Afro lepi & Caesalpinioideae & 0,31 & 1,11 & 2,1 & 0,97 & 9,7 \\
\hline $\begin{array}{l}\text { Beilschmiedia congolana } \\
\text { Robyns \& R. Wilczek }\end{array}$ & Beilcong & Lauraceae & 0,34 & $-0,95$ & 1,7 & 0,86 & $-9,1$ \\
\hline $\begin{array}{l}\text { Dacryodes klaineana } \\
\text { (Pierre) H.J. Lam }\end{array}$ & Dacrklai & Burseraceae & 0,31 & 1,11 & 2,1 & 0,97 & 9,7 \\
\hline Fillaeopsis discophora Harms & Fill disc & Fabaceae & 0,35 & $-0,91$ & 1,6 & 0,82 & $-8,9$ \\
\hline Celtis tessmannii Rendle & Celttesm & Ulmaceae & 0,34 & $-0,95$ & 1,7 & 0,86 & $-9,1$ \\
\hline Treculia africana Desc. & Trecafri & Moraceae & 0,34 & $-0,95$ & 1,7 & 0,86 & $-9,1$ \\
\hline Drypetes gossweileri S. Moore & Drypgoss & $\begin{array}{l}\text { Euphorbiaceae/ } \\
\text { Putranjivaceae }\end{array}$ & 0,31 & 1,11 & 2,1 & 0,97 & 9,7 \\
\hline $\begin{array}{l}\text { Eriocoelum macrocarpum } \\
\text { Gilg ex Engl. et Radlk. }\end{array}$ & Eriomacr & Sapindaceae & 0,31 & 1,07 & 2,0 & 0,94 & 9,5 \\
\hline $\begin{array}{l}\text { Piptadeniastrum africanum } \\
\text { (Hook. f.) Brenan }\end{array}$ & Piptafri & Mimosaceae & 0,31 & 1,11 & 2,1 & 0,97 & 9,7 \\
\hline Dialium pachyphyllum Harms & Dial pach & $\begin{array}{l}\text { Caesalpiniaceae } \\
\text { (Fabaceae) }\end{array}$ & 0,31 & 1,11 & 2,1 & 0,97 & 9,7 \\
\hline $\begin{array}{l}\text { Dacryodes buettneri } \\
\text { (Engl.) H.J. Lam }\end{array}$ & Dacrbutt & Burseraceae & 0,34 & $-0,95$ & 1,7 & 0,86 & $-9,1$ \\
\hline $\begin{array}{l}\text { Pancovia pedicellaris } \\
\text { Radlk. \& Gilg }\end{array}$ & Pancpedi & Sapindaceae & 0,31 & 1,11 & 2,1 & 0,97 & 9,7 \\
\hline Strombosiopsis tetrandra Engl & Stroteta & Olacaceae & 0,34 & $-0,95$ & 1,7 & 0,86 & $-9,1$ \\
\hline Strombosia pustulata Oliv. & Stropust & Olacaceae & 0,31 & 1,11 & 2,1 & 0,97 & 9,7 \\
\hline Heisteria parvifolia Sm. & Heisparv & Olacaceae & 0,31 & 1,11 & 2,1 & 0,97 & 9,7 \\
\hline $\begin{array}{l}\text { Campostylus mannii } \\
\text { (Oliver) Gilg }\end{array}$ & Camp mann & Achariaceae & 0,31 & 1,11 & 2,1 & 0,97 & 9,7 \\
\hline Chytranthus angustifolius Exell & Chytangu & Sapindaceae & 0,31 & 1,11 & 2,1 & 0,97 & 9,7 \\
\hline Diospyros hoyleana F. White & Dioshoyl & Ebenaceae & 0,31 & 1,11 & 2,1 & 0,97 & 9,7 \\
\hline $\begin{array}{l}\text { Diospyros conocarpa } \\
\text { Gürke \& K. Schum. }\end{array}$ & Dioscano & Ebenaceae & 0,31 & 1,11 & 2,1 & 0,97 & 9,7 \\
\hline $\begin{array}{l}\text { Synsepalum longecuneatum } \\
\text { De Wild. }\end{array}$ & Syns long & Sapotaceae & 0,31 & 1,11 & 2,1 & 0,97 & 9,7 \\
\hline Coelocaryon preussii Warb. & Coelpreu & Myristicaceae & 0,34 & $-0,95$ & 1,7 & 0,86 & $-9,1$ \\
\hline $\begin{array}{l}\text { Uapaca paludosa } \\
\text { Aubrév. \& Léandri }\end{array}$ & Uapa palu & $\begin{array}{l}\text { Euphorbiaceae/ } \\
\text { Phyllanthaceae }\end{array}$ & 0,34 & $-0,95$ & 1,7 & 0,86 & $-9,1$ \\
\hline $\begin{array}{l}\text { Irvingia gabonensis } \\
\text { (Aubry-Lecomte ex O'Rorke) } \\
\text { Baill. }\end{array}$ & Irvigabo & Irvingiaceae & 0,34 & $-0,95$ & 1,7 & 0,86 & 9,7 \\
\hline
\end{tabular}


Tableau IV.

Axe 2 : chablis multiples/complexes et espèces associées.

\begin{tabular}{|c|c|c|c|c|c|c|c|}
\hline Appellation/ variable & Abréviation & Famille & Poids & Coordonnées & Contribution & $\begin{array}{l}\text { Cosinus } \\
\text { carré }\end{array}$ & $\begin{array}{c}\text { Valeur } \\
\text { test }\end{array}$ \\
\hline Chablis multiple & $\mathrm{ChMu}$ & & 0,01 & $-6,06$ & 6,7 & 0,77 & $-8,6$ \\
\hline Chablis complexe & ChC & & 0,01 & $-6,00$ & 6,1 & 0,75 & $-8,5$ \\
\hline Musanga cecropioides R. Br. & Musa cerc & Cecropiaceae & 0,02 & $-4,76$ & 6,2 & 0,72 & $-8,3$ \\
\hline Psychotria gilletii De Wild. & Psycsp & Rubiaceae & 0,01 & $-3,15$ & 1,8 & 0,21 & $-4,5$ \\
\hline Octolobus spectabilis Welw. & Octospec & Malvaceae & 0,04 & $-1,89$ & 2,0 & 0,23 & $-4,8$ \\
\hline Berlinia spp. & Berlspp & $\begin{array}{c}\text { Caesalpiniaceae } \\
\text { (Fabaceae) }\end{array}$ & 0,05 & $-1,82$ & 2,1 & 0,26 & $-5,0$ \\
\hline Macaranga monandra Müll. Arg. & Macamona & Euphorbiaceae & 0,03 & $-3,21$ & 3,7 & 0,44 & $-6,5$ \\
\hline Macaranga barteri Müll. Arg. & Macabart & Euphorbiaceae & 0,01 & $-6,06$ & 6,7 & 0,77 & $-6,6$ \\
\hline Macaranga spinosa Müll. Arg. & maca spin & Euphorbiaceae & 0,04 & $-2,89$ & 3,8 & 0,45 & $-8,6$ \\
\hline Lindackeria dentata (Oliv.) Gilg & Lind dent & Achariaceae & 0,01 & $-6,06$ & 6,7 & 0,77 & $-8,6$ \\
\hline $\begin{array}{l}\text { Porterandia cladantha } \\
\text { (K. Schum.) Keay }\end{array}$ & Port clad & Rubiaceae & 0,05 & $-1,61$ & 1,6 & 0,20 & $-4,4$ \\
\hline Millettia mannii Baker & Mill mann & Fabaceae & 0,04 & $-2,33$ & 3,0 & 0,35 & $-5,9$ \\
\hline $\begin{array}{l}\text { Tetrapleura tetraptera } \\
\text { (Schumach. et Thonn) Taub. }\end{array}$ & Tetrtetr & Mimosaceae & 0,01 & $-4,09$ & 3,0 & 0,35 & $-5,8$ \\
\hline Calpocalyx dinklagei Harms & Cali & Fabaceae & 0,04 & $-2,19$ & 2,6 & 0,31 & $-5,5$ \\
\hline
\end{tabular}

Tableau V.

Axe 3 : chablis élémentaires et espèces associées.

\begin{tabular}{|c|c|c|c|c|c|c|c|}
\hline Appellation/variable & Abréviation & Famille & Poids & Coordonnées & Contribution & $\begin{array}{l}\text { Cosinus } \\
\text { carré }\end{array}$ & $\begin{array}{c}\text { Valeur } \\
\text { test }\end{array}$ \\
\hline Chablis élémentaire & ChE & & 0,03 & 4,17 & 8,8 & 0,74 & 8,5 \\
\hline Memecylon sp. & Meme & Melastomataceae & 0,03 & 2,85 & 4,1 & 0,34 & 5,8 \\
\hline $\begin{array}{l}\text { Corynanthe mayumbensis } \\
\text { (R.D. Good) Raym.-Hamet }\end{array}$ & Cory mayu & Rubiaceae & 0,06 & 2,48 & 6,2 & 0,55 & 7,3 \\
\hline $\begin{array}{l}\text { Dacryodes normandii Aubrév. } \\
\text { et Pellegr. }\end{array}$ & Dacrnorm & Burseraceae & 0,05 & 1,20 & 1,3 & 0,11 & 3,3 \\
\hline $\begin{array}{l}\text { Hymenostegia pellegrini } \\
\text { (A. Chev.) Léonard }\end{array}$ & Hymepell & Caesalpiniaceae & 0,14 & 0,86 & 1,8 & 0,18 & 4,1 \\
\hline $\begin{array}{l}\text { Blighia welwitschii } \\
\text { (Hiern) Radlk. }\end{array}$ & Bligwelw & Sapindaceae & 0,02 & 1,85 & 1,3 & 0,11 & 3,2 \\
\hline Pancovia sp. B & Pancspp & Sapindaceae & 0,16 & 0,84 & 2,0 & 0,21 & 4,5 \\
\hline Corynanthe johimbe K. Schum. & Pausjohi & Rubiaceae & 0,09 & 0,85 & 1,1 & 0,10 & 3,1 \\
\hline Centroplacus glaucinus Pierre & Cent gl & Pandaceae & 0,20 & 0,67 & 1,6 & 0,18 & 4,2 \\
\hline $\begin{array}{l}\text { Tabernaemontana crassa } \\
\text { G. Bentham }\end{array}$ & Tabecras & Apocynaceae & 0,03 & 2,56 & 3,3 & 0,28 & 5,2 \\
\hline Erismadelphus exsul Mildbr. & Erisexcs & Vochysiaceae & 0,02 & 4,05 & 6,2 & 0,52 & 7,1 \\
\hline $\begin{array}{l}\text { Trichoscypha abut Engler } \\
\text { et V. Brehm }\end{array}$ & Tric abut & Anacardiaceae & 0,03 & 3,92 & 7,7 & 0,65 & 8,0 \\
\hline Coelocaryon klainei Pierre & Coelklai & Myristicaceae & 0,02 & 4,25 & 6,8 & 0,57 & 7,4 \\
\hline
\end{tabular}


Léandri, Irvingia gabonensis (Aubry-Lecomte ex O’Rorke) Baill. Le deuxième axe discrimine les chablis complexes, multiples et les espèces ci-après qui s'y régénèrent (tableau IV) : Musanga cecropioides R. Br., Psychotria gilletii De Wild., Octolobus spectabilis Welw., Berlinia spp., Macaranga monandra Müll. Arg., Macaranga barteri Müll. Arg., Macaranga spinosa Müll. Arg., Lindackeria dentata (Oliv.) Gilg, Porterandia cladantha (K. Schum.) Keay, Millettia mannii Baker, Tetrapleura tetraptera (Schumach. et Thonn) Taub. et Calpocalyx dinklagei Harms. Le troisième axe regroupe les chablis élémentaires et les espèces qui se régénèrent dans ce type de chablis, notamment (tableau V) Memecylon sp., Corynanthe mayumbensis (R.D. Good) Raym.-Hamet, Dacryodes normandii (Aubrév.) Pellegr., Hymenostegia pellegrini (A. Chev.) Léonard, Blighia welwitschii (Hiern) Radlk., Pancovia sp. B, Corynanthe johimbe K. Schum., Centroplacus glaucinus Pierre, Tabernaemontana crassa G. Bentham, Erismadelphus exsul Mildbr., Trichoscypha abut (Engler) V. Brehm et Coelocaryon klainei Pierre. Le plan factoriel le plus expressif (figure 3), constitué par les axes 1 (18,19\% de la variance totale) et 2 ( $7,83 \%)$, fait principalement apparaître une attraction entre les espèces héliophiles, les chablis complexes et les chablis multiples (axe 2). Trois groupes d'espèces liées aux chablis partiels de petite taille montrent une opposition entre espèces d'arbres.

Les résultats montrent que la régénération de 47 espèces est associée aux différents types de chablis. La majorité de ces espèces de chablis (environ $49 \%$ ) se régénèrent dans les chablis partiels ; $26 \%$ dépendent des chablis complexes et multiples ; $25 \%$ établissent leur régénération dans les chablis élémentaires. Cependant, une grande partie des espèces recensées (74\% du total) ne semblent pas avoir de lien manifeste avec les chablis. Les observations de terrain montrent que ces espèces sont peu fréquentes dans les chablis. Elles sont présentes de manière erratique, dans un nombre réduit de chablis, avec de très faibles densités, souvent un seul ou deux individus.

\section{Discussion}

\section{Régime de perturbations par chablis de la forêt d'Ipassa}

Le régime de perturbations de la forêt d'Ipassa est principalement caractérisé par une dynamique de chablis de faibles dimensions (chablis partiels et chablis élémentaires). Les chablis constitués par la mort de plusieurs arbres qui créent de grandes trouées sont moins fréquents. Ces résultats concordent avec ceux obtenus dans le Parc national de Korup au Cameroun (Egbe et Moura, 2012). Les petits chablis sont aussi plus fréquents que les grands chablis dans d'autres forêts tropicales (Tabarelli et Mantovani, 2000 ; Martins et Rodrigues, 2002). Nous avons également trouvé que la taille moyenne des trouées sur ce site variait de $52 \mathrm{~m}^{2}$ à $625 \mathrm{~m}^{2}$. Florence (1981), dans la même forêt, avait obtenu une surface moyenne des chablis relativement similaire, allant de $120 \mathrm{~m}^{2}$ à $400 \mathrm{~m}^{2}$. Nos résultats restent dans le même ordre de grandeur que les valeurs observées dans d'autres forêts tropicales. Brokaw (1985) au Panama a trouvé

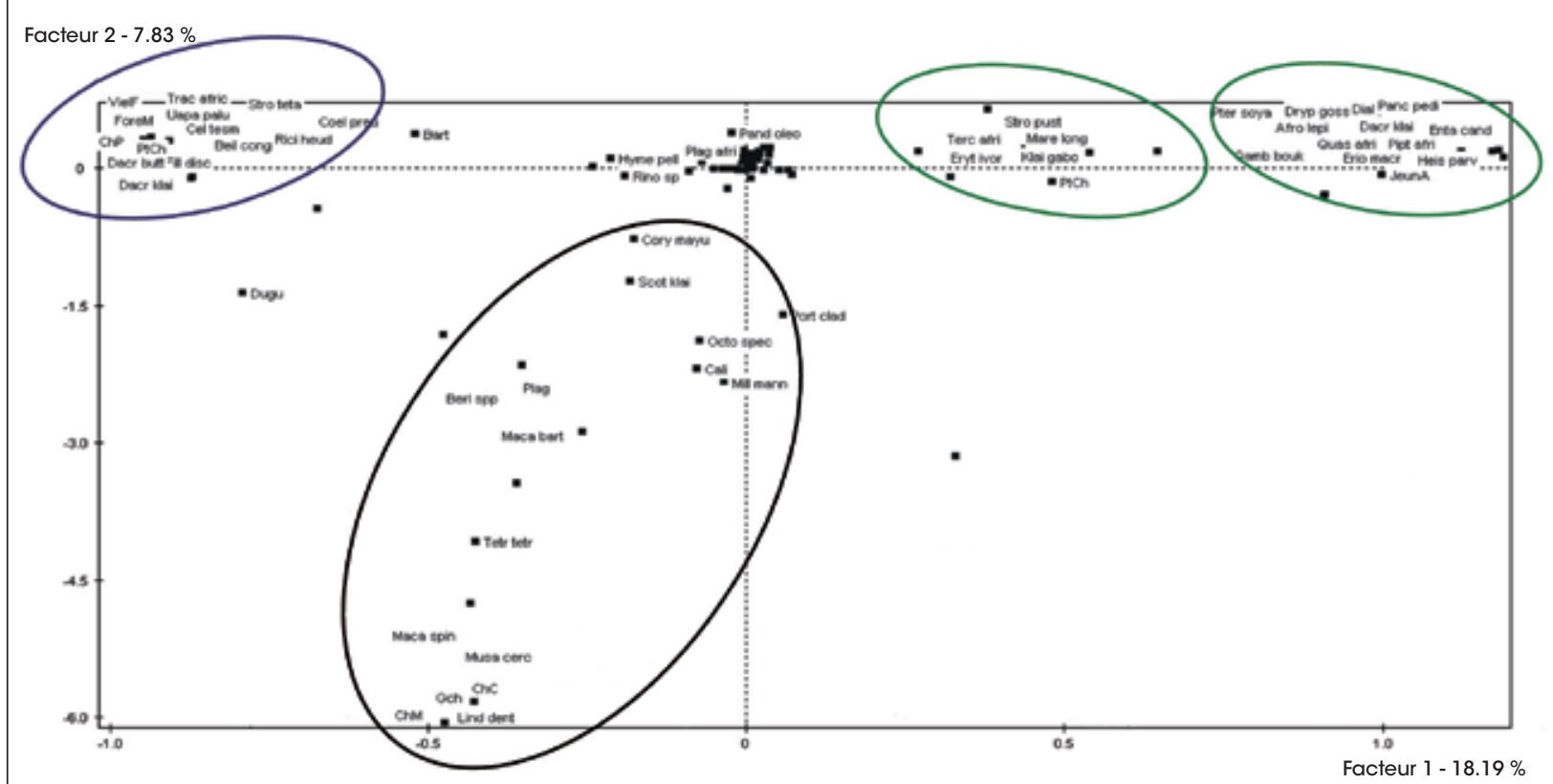

Figure 3.

Analyse de correspondances multiples, les deux premiers axes factoriels résumant l'effet des chablis. Les abréviations figurent sur les tableaux III, IV et V. 
$20 \mathrm{~m}^{2}$ à $705 \mathrm{~m}^{2}$ alors que Gale (2000) en Équateur et Lima et al. (2008) au Brésil ont respectivement relevé des surfaces moyennes de chablis de 150 à $1100 \mathrm{~m}^{2}$ et 121 à $333 \mathrm{~m}^{2}$. La dimension des trouées est souvent influencée par de multiples facteurs tels que l'abondance des lianes, les causes des chutes, le nombre et la taille des arbres tombés, les stades de développement de la forêt (Yamamoto, 2000 ; Lima et Moura, 2008) ainsi que les facteurs climatiques (Sapkota et Odén, 2009). D’autres études plus approfondies sont nécessaires pour comprendre ces interrelations. Par ailleurs, nous n'avons pas observé de nouveaux chablis qui se superposent à ceux déjà recensés. Il semble pourtant que les zones de forêts touchées par un chablis deviennent fragiles et sont plus susceptibles d'être le théâtre de nouveaux chablis (Riera et Alexandre, 1988), en raison de "l'effet de rive » décrit par Oldeman (1972). Une période d'observation beaucoup plus longue aurait peut-être permis de percevoir ce type de phénomène.

Nos résultats montrent en outre que les chablis affectent annuellement environ 1,3\% de la surface forestière. Les calculs ont aussi donné, pour une période de 100 ans, un pourcentage annuel de chablis de $4 \%$. Ces valeurs sont proches des moyennes obtenues dans d'autres forêts tropicales. Arihafa et Mack (2013) en Papouasie-Nouvelle-Guinée rapportent une proportion annuelle de forêt couverte par les chablis s'élevant à 1,5 \%. En Guyane française, Riera (1983) a noté une proportion annuelle de chablis de 1,1\%.

Nous avons par ailleurs estimé la demi-vie à 53 ans. Florence (1981) avait également relevé dans cette forêt un temps de renouvellement très rapide de 60 ans. Le temps de renouvellement sur le site d'Ipassa semble très court et serait associé à l'effet du vent. En effet, la forêt étudiée se situe sur un plateau qui surplombe le grand fleuve Ivindo. Selon Hladik et Blanc (1987), des orages souvent très violents arrivent par la vallée de ce fleuve large de plus d'un kilomètre. De ce fait, la forêt est souvent assujettie à des vents violents et semble plus perturbée dans les parties

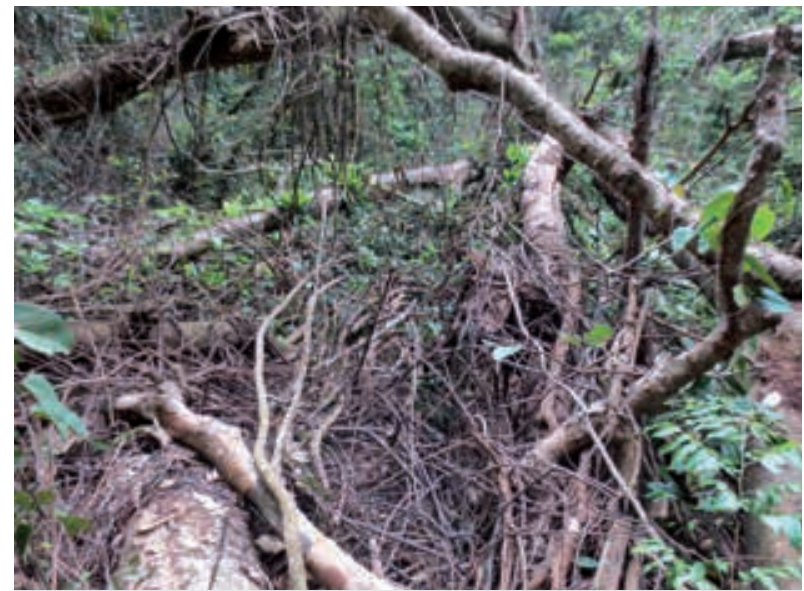

Photo 3.

Chablis multiple.

Photo J. P. Obame Engone. proches du bord du plateau. À l'opposé, le temps de renouvellement paraît plus long dans d'autres forêts tropicales, soit environ 60 à 240 ans en Amérique centrale (Hunter et al., 2015), 75 à 417 ans en forêt dense humide sempervirente de Taï en Côte d'Ivoire (Bonnis, 1980) et 97 ans au Mexique (Torquebiau, 1981). Il aurait été intéressant d'analyser les comportements des chablis en lien avec les facteurs climatiques. Cependant, l'influence du climat local sur les chablis n'avait pas été directement mesurée dans cette étude.

\section{Effet des types de chablis sur les espèces d'arbres}

Selon Hubbell et al. (1999), la composition spécifique des chablis est imprévisible, même pour les espèces pionnières, en raison de la limitation en dispersion. Pour Barton (1984), Brown et Whitmore (1992), Kennedy et Swaine (1992) et Clark et al. (1993), les espèces d'arbres ne montrent pas de préférence pour s'établir dans des trouées de différentes tailles générées par les chablis. Cependant, nous avons identifié 35 espèces d'arbres qui se régénèrent préférentiellement dans les chablis causés par la chute de grosses branches, de couronnes d'arbres ou d'un seul arbre. Dans la littérature scientifique consultée (Normand et Paquis, 1976 ; Letouzey, 1983 ; Villiers, 1989 ; Tailfer, 1989), ces espèces font partie de la canopée ou du sous-étage et sont décrites comme tolérantes à l'ombrage. Nous savions déjà que les ouvertures créées par les chablis étaient déterminantes pour la régénération et le maintien d'espèces pionnières dans les peuplements forestiers (Hartshorn, 1980 ; Alexandre, 1982 ; Brokaw, 1985 ; Brokaw et Scheiner, 1989 ; Jans et al., 1993 ; Flores et al., 2006). La présente étude démontre de surcroît que la régénération de plusieurs espèces d'arbres tolérantes à l'ombrage se produit dans les chablis partiels et les chablis élémentaires. Ces deux types de chablis semblent d'ailleurs constituer l'un des mécanismes permettant à plusieurs espèces d'arbres de la canopée de s'insérer et se maintenir

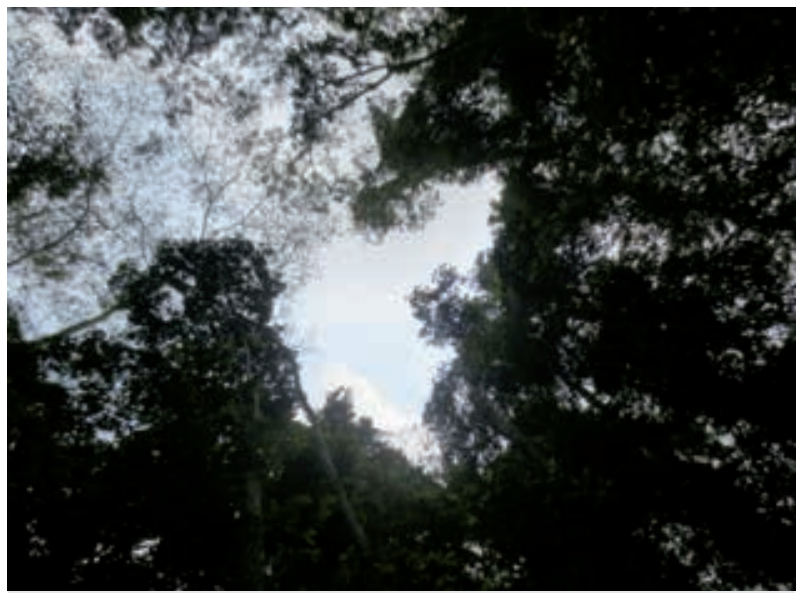

Photo 4.

Ouverture de la canopée par la chute d'une branche. Photo J. P. Obame Engone. 
dans les peuplements forestiers. Plusieurs études ont aussi souligné que les espèces tolérantes à l'ombrage colonisaient les chablis de faibles dimensions (Runkle, 1985 ; Laska, 2001 ; Schnitzer et al., 2008 ; Sapkota et Odén, 2009 ; Gravel et al., 2010). Pour Martins et Rodrigues (2002), la prédominance des petits chablis peut induire la dominance d'espèces tolérantes à l'ombrage dans les peuplements forestiers. Dans le même sens, nos résultats suggèrent que le régime de perturbations d'Ipassa dominé par les petits chablis pourrait influencer la composition forestière au bénéfice d'espèces tolérantes à l'ombrage. Des recherches plus approfondies devront évaluer la probabilité d'une telle relation. L'étude a également relevé une gamme d'espèces pionnières (Musanga cecropioides R. Br., Psychotria gilletii De Wild., Macaranga barteri Müll. Arg.) qui se régénèrent seulement dans les chablis multiples. Kahn (1982) avait aussi recensé ces mêmes arbres dans les milieux perturbés en Côte d'Ivoire. La dépendance des espèces héliophiles aux chablis est bien connue (Brokaw, 1987 ; Brokaw et Busing, 2000 ; Yamamoto, 2000), à la seule différence qu'ici le phénomène ne semble pas généralisé. L'étude montre en effet que les chablis complexes et multiples (auxquels sont associées les espèces héliophiles) ne constituent que $30 \%$ du total des chablis recensés. Comme le soutiennent Becker et al. (1988), puis Montgomery et Chazdon (2002), la répartition des espèces entre types de chablis proviendrait des différences de besoin en lumière entre les espèces pionnières et les espèces tolérantes à l'ombrage.

L'étude relève enfin que plusieurs espèces recensées (74 \%) n'ont pas de liens manifestes avec les chablis. Cela suggère qu'en dehors des chablis, d'autres facteurs agissent sur la régénération de ces arbres. Plusieurs études indiquent en effet que des processus intrinsèques à l'écosystème comme la limitation en dispersion peuvent influencer la présence des espèces d'arbres dans un milieu (Hubbell et Borda-de-Água, 2004 ; Etienne et Alonso, 2006 ; May et al., 2012 ; Rosindell et al., 2011). Il est aussi possible que notre étude n'ait pas intégré certains facteurs qui contrôlent la régénération et le maintien des arbres.

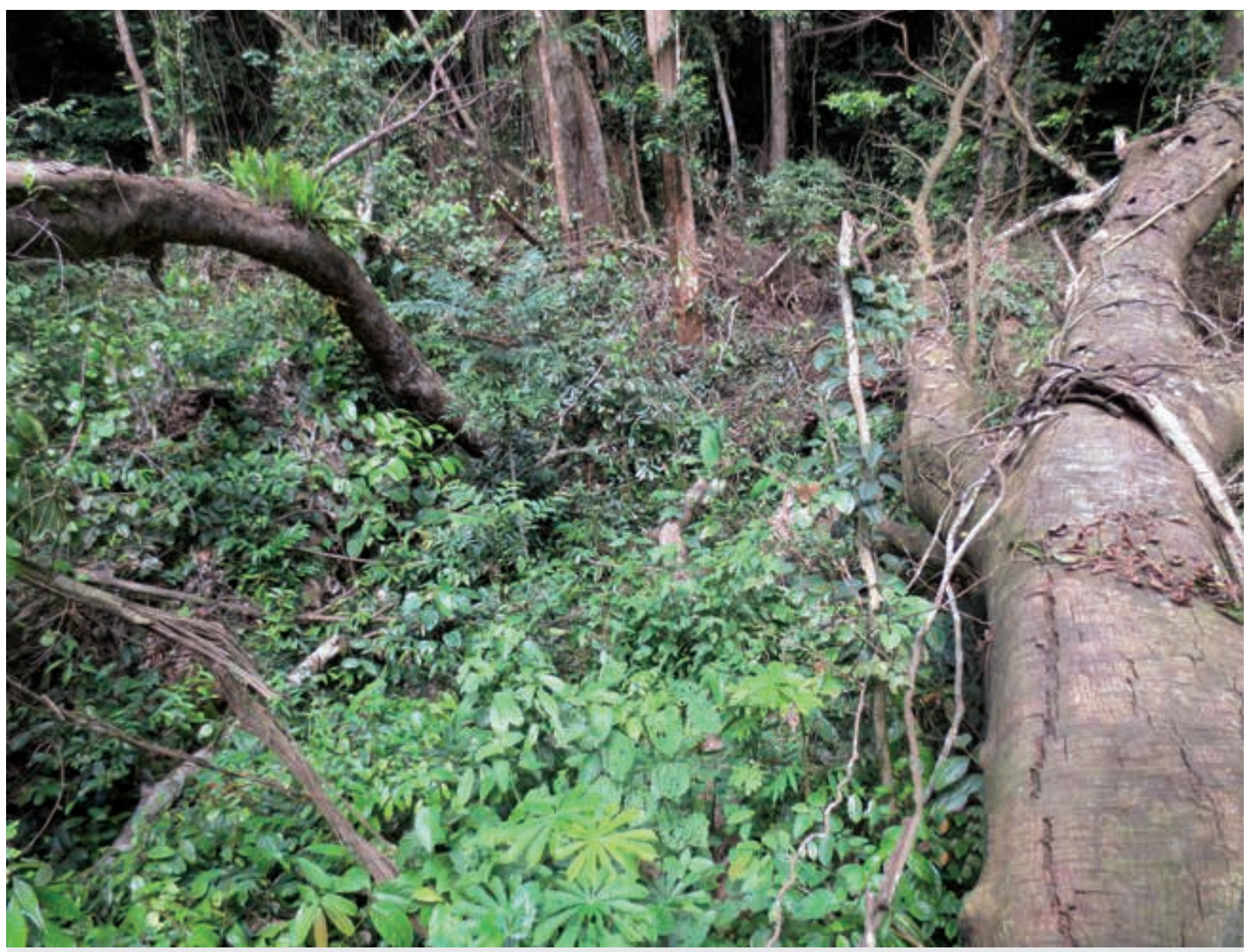

Photo 5.

Régénération naturelle de Musanga cecropioides (espèce héliophile) dans une trouée de chablis multiple. Photo J. P. Obame Engone. 


\section{Conclusion}

Notre étude a mis en évidence le régime de perturbations de la forêt d'Ipassa caractérisé par la dominance des chablis partiels et des chablis élémentaires. Ces deux types de chablis semblent aussi gouverner la régénération naturelle des essences tolérantes à l'ombrage qui peuplent la canopée et le sous-étage. Ce constat amène à penser que ces chablis offrent des conditions écologiques favorables pour ces espèces. Une meilleure compréhension des processus en œuvre dans ces milieux nécessite des recherches approfondies. On note également dans cette forêt la présence de chablis complexes et chablis multiples desquels dépendent les espèces héliophiles, qui ne peuvent se maintenir dans les peuplements forestiers qu'en se régénérant dans ces milieux. Par ailleurs, en comparaison avec d'autres forêts tropicales, la forêt étudiée présente un temps de renouvellement très court. Il conviendrait de comprendre les causes de ce phénomène. Il paraît enfin indispensable d'étudier sur ce site les aspects que notre étude n'a pas pu intégrer, notamment le recrutement, les accroissements, la mortalité des jeunes plants au sein des différents types de trouées ainsi que la limitation en dispersion.

\section{Références bibliographiques}

Alexandre D. Y., 1982. Aspects de la régénération naturelle en forêt dense de Côte d'Ivoire. Candollea, 37 : 579-588.

Arihafa A., Mack A. L., 2013. Treefall Gap Dynamics in a Tropical Rain Forest in Papua New Guinea. Pacific Science, 67 (1): 47-58.

ATIBT-FFEM, 2014. Étude sur le plan pratique d'aménagement des forêts naturelles de production tropicales africaines. Volet 4. Gestion durable et préconisations en vue de la certification. Sainte-Ode, Belgique, Impribeau, 162 p.

Barton A. M., 1984. Neotropical pioneer and shadetolerant tree species: do they partition treefall gaps? Tropical Ecology, 25: 196-202.

Battles J. J., Dushoff J. G., Fahey T. J., 1996. Line intersect sampling of forest canopy gaps. Forest Science, 42: 131-138.

Beaujour A., 1971. Notice explicative, Makokou-Ouest et carte géologique de reconnaissance au 1/50 000. Ministère des Mines, des Ressources Hydrauliques et de l'Énergie, Gabon.

Becker P., Rabenold P. E., Idol J. R., Smith A. P., 1988. Water potential gradients for gaps and slopes in a Panamanian tropical moist forests dry season. Journal of Tropical Ecology, 4 (2): 173-184.

Benzécri J.-P., 1973. L'Analyse des données. 2. L'Analyse des correspondances. Paris, France, Dunod, $619 \mathrm{p}$.

Bonnis G., 1980. Étude des chablis en forêt dense humide sempervirente naturelle de Taï (Côte d'Ivoire). Abidjan,
Côte d'Ivoire, Office de la recherche scientifique et technique outre-mer, $68 \mathrm{p}$.

Brokaw N. V. L., 1985. Gap-phase regeneration in a tropical forest. Ecology, 66: 682-687.

Brokaw N. V. L., 1987. Gap-phase regeneration of three pioneer tree species in a tropical forest. Journal of Ecology, 75: 9-19.

Brokaw N. V. L., Scheiner S. M., 1989. Species composition in gaps and structure of a tropical forest. Ecology, 70: 538-541.

Brokaw N., Busing R. T., 2000. Niche versus chance and tree diversity in forest gaps. Trends in Ecology and Evolution, 15: $183-188$.

Brown N. D., Whitmore T. C., 1992. Do dipterocarp seedlings really partition tropical rain forest gaps? Philosophical Transactions of the Royal Society of London, series B, 335: 369-378.

Cabalé G., 1978. Essai phytogéographique sur la forêt dense du Gabon. Annales de l'Université nationale du Gabon, série Sciences et Techniques, 2 : 87-101.

Cabalé G., Fontès J., 1978. Les inventaires forestiers au Gabon : applications à la phytogéographie. Bois et Forêts des Tropiques, 178 : 15-33. [En ligne] http://bft.cirad.fr/cd/ BFT_177_15-33.pdf

Chapman C. A., Chapman L. J., Kaufman L., Zanne A. E., 1999. Potential causes of arrested succession in Kibale National Park, Uganda: growth and mortality of seedlings. African Journal of Ecology, 37: 81-92.

Chazdon R. L., Fetcher N., 1984. Photosynthetic light environments in a lowland tropical rainforest in Costa Rica. Journal of Ecology, 72: 552-564.

Chesson P., 2000a. General theory of competitive coexistence in spatially-varying environments. Theoretical Population Biology, 58: 211-237.

Chesson P., 200ob. Mechanisms of maintenance of species diversity. Annual Review of Ecology and Systematics, 31: 343-366.

Cibois P., 2007. Les méthodes d'analyse d'enquêtes. Paris, France, PUF, « Que sais-je ? », n³782, 128 p.

Clark D. A., Clark D. B., 1992. Life history diversity of canopy and emergent trees in a neotropical rain forest. Ecological Monographs, 62: 315-344.

Clark D. B., Clark D. A., Rich P. M., 1993. Comparative analysis of microhabitat utilization by saplings of nine tree species in neotropical rain forest. Biotropica, 25: 397-407.

Denslow J. S., 1980. Patterns of plant species diversity during succession under different disturbance regimes. Ecologia, 46: 18-21.

Denslow J. S., 1980. Gap partitioning among tropical rain forest trees. Biotropica, 12 (supplement): 47-55.

Denslow J. S., 1987. Tropical rainforest gaps and tree species diversity. Annual Review of Ecology and Systematics, 18: 431-451. 
Denslow J. S., Ellison A., Sanford Jr R. E., 1998. Treefall gap size effects on ecological processes in a tropical wet forest. Journal of Ecology, 86: 597-609.

Duveiller G., Defourny P., Desclée B., Mayaux P., 2008. Deforestation in Central Africa: estimates at regional, national and landscape levels by advanced processing of systematically distributed Landsat extracts. Remote Sensing and Environment, 112 (5): 1969-1981.

Drouineau S., Nasi R., 1999. L'aménagement forestier au Gabon : historique, bilan, perspectives. Montpellier, France, Cirad-Forêt, série Forafri 19, 64 p.

Egbe E. A., Chuyong G. B., Fonge B. A., Namuene K. S., 2012. Forest disturbance and natural regeneration in an African rainforest at Korup National Park, Cameroon. International Journal of Biodiversity and Conservation, 4 (11): 377-384.

Escofier B., Pagès J., 2008. Analyses factorielles simples et multiples. Objectifs, méthodes et interprétation. Paris, France, Dunod, $318 \mathrm{p}$.

Etienne R. S., Alonso D., 2007. Neutral community theory: how stochasticity and dispersal-limitation can explain species coexistence. Journal of Statistical Physics, 128 (1/2): 485-510.

Florence J., 1981. Chablis et sylvigenèse dans une forêt dense humide sempervirente du Gabon. Thèse de $3^{\mathrm{e}}$ cycle, Université Louis Pasteur, Strasbourg, France, 261 p.

Flores O., Gourlet-Fleury S., Picard N., 2006. Local disturbance, forest structure and dispersal effects on sapling distribution of light-demanding and shade-tolerant species in a French Guianian forest. Acta Ecologica, 29: 141-154.

Frontier S., 1976. Utilisation des diagrammes rang-fréquence dans l'analyse des écosystèmes. Journal de Recherche Océanographique, 1 (3) : 35-48.

Gale N., 2000. The relationship between canopy gaps and topography in a Western Ecuadorian rain forest. Biotropica, 32: 653-661.

Gravel D., Canham C. D., Beaudet M., Messier C., 2010. Shade tolerance, canopy gaps and mechanisms of coexistence of forest trees. Oikos, 119: 475-484.

Hladik A., Blanc P., 1987. Croissance des plantes en sousbois de forêt dense humide (Makokou, Gabon). Revue d'Écologie, Terre et Vie, 42 : 209-234.

Hartshorn G. S., 1978. Treefall and tropical forest dynamics. In: Tomlinson P. B., Zimmermann M. H. (eds). Tropical Trees as Living Systems. Londres, Royaume-Uni, Cambridge University Press, 617-638.

Hartshorn G. S., 1980. Neotropical forest dynamics. Biotropica, 12 (supplement): 23-30.

Hubbell S. P., Foster R. B., O’Brien S. T., Harms K. E., Condit R., Wechsler B., Wright S. J., Loo de Lao S., 1999.
Light-gap disturbances, recruitment limitation and tree diversity in a Neotropical Forest. Science, 283: 554-557.

Hubbell S. P., Borda-de-Água L., 2004. The unified neutral theory of biodiversity and biogeography: reply. Ecology, 85 (11): 3175-3178.

Hunter M. O., Keller M., Morton D., Cook B., Lefsky M., Ducey M., Saleska S., de Oliveira R.C. Jr, Schietti J., 2015. Structural Dynamics of Tropical Moist Forest Gaps. PLoS ONE, 10 (7): e0132144. Doi:10.1371/journal.pone.0132144

Iret-Ecotrop, Unesco, 1987. Makokou, Gabon. Une station de recherche en écologie tropicale. Paris, France, Unesco, 55 p.

Jackson D., 1993. Stopping rules in Principal Components Analysis: A comparison of heuristical and statistical approaches. Ecology, 74 (8): 2204-2214.

Jans L., Poorter L., van Rompaey R., Bongers F., 1993. Gaps and forest zones in tropical moist forest in Ivory Coast. Biotropica, 25: 258-269.

Kabakoff R. P., Chazdon R. L., 1996. Effects of canopy species dominance on understory light availability in tropical secondary rain forest. Journal of Tropical Ecology, 12: 779-788.

Kahn F., 1982. La reconstitution de la forêt tropicale humide. Sud-Ouest de la Côte d'Ivoire. Paris, France, Orstom, coll. Mémoires n97, 150 p.

Kennedy D. K., Swaine M. D., 1992. Germination and growth of colonizing species in artificial gaps of different sizes in dipterocarp rain forest. Philosophical Transactions of the Royal Society, series B, 335: 357-367.

Laurance W., Alonso A., Lee M., Campbell P., 2006. Challenges for forest conservation in Gabon, Central Africa. Futures, 38: 454-470.

Laska G., 2001. The disturbance and vegetation dynamics: a review and an alternative framework. Plant Ecology, 157: 77-99.

Lebart L., Piron M., Morineau A., 2006. Statistique exploratoire multidimensionnelle. Paris, France, Dunod, $464 \mathrm{p}$.

Legendre P., Legendre L., 2012. Numerical Ecology. $3^{\text {rd }}$ ed. Amsterdam, Pays-Bas, Elsevier Science BV, 990 p.

Letouzey R., 1983. Manuel de botanique forestière. Afrique tropicale. Tome 2 B. Centre Technique Forestier Tropical (Cirad), Nogent sur Marne, France, $461 \mathrm{p}$.

Lima R. A. F., Moura L. C., 2008. Gap disturbance regime and composition in the Atlantic Montane Rain Forest: the influence of topography. Plant Ecology, 197: 239-253.

Martins S. V., Rodrigues R. R., 2002. Gap-phase regeneration in a semideciduous mesophytic forest, south-eastern Brazil. Plant Ecology, 163: 51-62.

May F., Giladi I., Yaron Ziv Y., Florian Jeltsch F., 2011. Dispersal and diversity-unifying scale-dependent relationships within the neutral theory. Oikos, 121: 942-951. 
Montgomery R. A., Chazdon R. L., 2002. Light gradient partitioning by tropical tree seedlings in the absence of canopy gaps. Ecologia, 131: 165-174.

Mutoji-A-Kazadi, 1977. Notes de sylvigénèse pour la Guyane : transect et photographies aériennes. DEA, Université des sciences et techniques du Languedoc, Montpellier, France, $35 \mathrm{p}$.

Normand D., Paquis J., 1976. Manuel d'identification des bois commerciaux. Tome 2. Afrique guinéo-congolaise. Nogentsur-Marne, France, Centre technique forestier tropical, $335 \mathrm{p}$.

Oldeman R. A. A., 1972. L'architecture de la végétation ripicole forestière des fleuves et criques guyanais. Adansonia, série 2, 12 (2) : 253-265.

Oldeman R. A. A., 1979. Quelques aspects quantifiables de l'arborigenèse et de la sylvigenèse. Fcologica Plantarum, $14: 289-312$.

Oldeman R. A. A., 1989. Dynamics in tropical rain forests. In: Tropical Forests: Botanical Dynamics, Speciation and Diversity. Londres, Royaume-Uni, Academic Press Limited, 4-21.

Orians G. H., 1982. The influence of tree falls in tropical forests on tree species richness. Tropical Ecology, 23: 255-279.

Rao P., Barik S. K., Pandey H. N., Tripathi R. S., 1997. Tree seed germination and seedling establishment in tree falls gaps and understorey in a subtropical forest of northeast India. Australian Journal of Ecology, 22: 136-145.

Richards P. W., 1996. The Tropical Rain Forest. An Ecological Study. $2^{\text {nd }}$ ed. Cambridge, Royaume-Uni, Cambridge University Press.

Riera B., 1983. À propos des chablis en forêt guyanaise : piste de Saint-Elie. Bulletin Ecerex, 6 (7) : 214-225.

Riera B., Alexandre D.-Y., 1985. Les chablis : le calcul de la dynamique forestière en Guyane Française. Cayenne, France, Orstom, 7 p.

Riera B., Alexandre D.-Y., 1988. Surface des chablis et temps de renouvellement en forêt dense tropicale. Acta Ecologica, 9 (2) : 211-220.

Riera B., Puig H., Lescure J.-P., 1988. La dynamique de la forêt naturelle. Bois et Forêts des Tropiques, 219 : 69-78. [En ligne] http://bft.cirad.fr/cd/BFT_219_69-78.pdf

Rollet B., 1969. La régénération en forêt dense humide sempervirente de plaine de la Guyane vénézuélienne. Bois et Forêts des Tropiques, 124 : 19-38. [En ligne] http://bft.cirad. $\mathrm{fr} / \mathrm{cd} /$ BFT_124_19-38.pdf

Rollet B., 1974. L'architecture des forêts denses humides sempervirentes de plaine. Nogent-sur-Marne, France, Centre technique forestier tropical, $298 \mathrm{p}$.

Rollet B., 1983. La régénération naturelle dans les trouées. Bois et Forêts des Tropiques, 201 : 3-34. [En ligne] http://bft. cirad.fr/cd/BFT_201_3-34.pdf

Rosindell J., Hubbell S. P., Etienne R. S., 2011. The Unified Neutral Theory of Biodiversity and Biogeography at Age Ten. Trends in Ecology and Evolution, 26 (7): 340-348.
Runkle J. R., 1981. Gap regeneration in some old-growth forests of the eastern United States. Ecology, 62: 1041-1051.

Runkle J. R., 1985. Comparison of methods for determining fraction of land area in treefall gaps. Forest Science, 31: $15-19$.

Runkle J. R., 1992. Guidelines and sample protocol for sampling forest gaps. USDA Forest Service, Pacific Northwest Research Station, 44 p.

Saporta G., 2006. Probabilités, analyse des données et statistique. Paris, France, Éd. Technip, 622 p.

Sapkota I. P., Odén P. C., 2009. Gap characteristics and their effects on regeneration, dominance and early growth of woody species. Journal of Plant Ecology, 2 (1): 21-29.

Schnitzer S. A., Carson W. P., 2001. Treefall gaps and the maintenance of species diversity in a tropical forest. Ecology, 82 (4): 913-919.

Schnitzer S. A., Mascaro J., Carson W. P., 2008. Tropical forest community ecology. Oxford, Royaume-Uni, Wiley-Blackwell, $536 \mathrm{p}$.

Sherman R. E., Fahey T. J., Battles J. J., 2000. Smal-scale disturbance and regeneration dynamics in a neotropical mangrove forest. Journal of Ecology, 88: 165-178.

Tabarelli M., Mantovani W., 2000. Gap-phase regeneration in a tropical montane forest: the effects of gap structure and bamboo species. Plant Ecology, 148: 149-155.

Tailfer Y., 1989. La forêt dense d'Afrique centrale : identification pratique des principaux arbres. Tome 1 : Approche forestière et morphologique. Wageningen, Pays-Bas, CTA, 456 p.

Torquebiau E., 1981. Analyse architecturale de la forêt de Los Tuxtlas (Veracruz), Mexique. Université des sciences et techniques du Languedoc, Montpellier, France, $185 \mathrm{p}$.

Van Kekem A. J., 1984. Étude pédologique de la Réserve de la Biosphère de M'passa, Makokou, Gabon. Paris, France, Unesco, Programme Man and Biosphere (MAB), 34 p.

Villiers J.-F., 1989. Leguminosae, Mimosoideae. Flore du Gabon, volume 31. Paris, France, Muséum national d’histoire naturelle, $185 \mathrm{p}$.

Whitmore T. C., 1989. Canopy gaps and the two major groups of forest trees. Ecology, 70 (3): 536-538.

Yamamoto S. I., 1992. The gap theory in forest dynamics. Botanical Magazine, 105: 375-380.

Yamamoto S. I., 2000. Forest gap dynamics and tree regeneration. Journal of Forest Research, 5: 223-229.

Zanne A. E., Chapman C. A., 2005. Diversity of woody species in forest, tree fall gaps, and edge in Kibale National Park, Uganda. Plant Ecology, 178: 121-139. 